\title{
ACOPLAMENTO DE CONVERSORES ESTÁTICOS COM MALHA DE CONTROLE E DISPOSITIVOS ELETROMAGNÉTICOS NÃO-LINEARES
}

\author{
Jorge Roel O.* \\ Patrick Kuo-Peng*
}

Nelson Sadowski*

Nelson J. Batistela*

\author{
João P. A. Bastos* \\ * GRUCAD/EEL/CTC/UFSC, C.P. 476, 88040-900, Florianópolis, SC, Brasil \\ jorge@grucad.ufsc.br
}

\begin{abstract}
A static converter with its control loop coupled to a non-linear electromagnetic device is studied. The electromagnetic device is modelled by two-dimensional finite element method and the static converter is studied in the state space. The converter and electromagnetic device equations are then coupled together and solved simultaneously. An application with a full bridge inverter is presented.
\end{abstract}

KEYWORDS: Electromagnetic devices, finite element method, circuit simulation, nonlinearity, control loops.

\section{RESUMO}

O presente artigo aborda o acoplamento de dispositivos eletromagnéticos não lineares com conversores estáticos operando com malha de realimentação. O dispositivo eletromagnético é modelado pelo método dos elementos finitos em duas dimensões e o conversor estático é estudado no espaço de estado. As equações do dispositivo eletromagnético, do conversor e do controle são acopla-

\footnotetext{
Artigo submetido em 20/12/00

1a. Revisão em 31/05/01

Aceito sob recomendação do Ed. Cons. Prof. Dr. Edson H. Watanabe
}

das e resolvidas simultaneamente. Uma aplicação com um inversor em ponte completa com uma malha de alimentação é apresentada.

\section{INTRODUÇÃO}

O comportamento dos dispositivos eletromagnéticos é caracterizado pela distribuição dos campos eletromagnéticos no núcleo magnético e na região circunvizinha. As equações de Maxwell regem estes campos eletromagnéticos. A solução destas equações, na maioria dos casos reais, é de difícil abordagem por métodos analíticos. Com o desenvolvimento dos computadores digitais, permitiu-se a utilização de métodos numéricos para a solução das equações de Maxwell e para descrever, de modo mais preciso, o comportamento destes dispositivos eletromagnéticos. Neste trabalho são apresentadas as equações que descrevem o comportamento de uma estrutura eletromagnética em duas dimensões. Estas equações são aproximadas pelo método residual de Galerkin. A discretização e resolução são feitas utilizando o método dos elementos finitos (Bastos, 1992), o qual é uma técnica numérica que parte do princípio de que o domínio de estudo deve ser decomposto ou discretizado em pequenas regiões, chamadas de elementos finitos. Após a aplicação do método de elementos finitos, obtém-se um sistema matricial de equações que descreve 
o comportamento da estrutura eletromagnética.

Na simulação de dispositivos eletromagnéticos alimentados por conversores estáticos, é utilizada uma resolução simultânea das equações de campo do dispositivo e do circuito do conversor. As equações de estado do conversor são determinadas para qualquer seqüência de operação durante a simulação, de modo automático, a partir da descrição topológica do circuito (Balabanian e Bickart, 1969). As equações de campo da estrutura eletromagnética são combinadas diretamente com as equações de estado do circuito do conversor. Este acoplamento é realizado através de duas grandezas comuns à estrutura eletromagnética e ao conversor, que são a corrente e a tensão nos terminais das estruturas eletromagnéticas.

Neste trabalho, adota-se o modelo resistivo binário para os interruptores do circuito de potência: quando o interruptor está aberto, tem-se uma resistência elétrica alta, e para o interruptor fechado, uma resistência baixa. Os semicondutores de potência comandados recebem sinais que são gerados por uma técnica apropriada de controle, que inclusive pode ser a malha fechada. O estudo do comportamento de uma estrutura de potência em malha fechada operando com dispositivos magnéticos na região não linear é de difícil modelagem e análise. O método aqui apresentado é uma possibilidade viável de se avaliar o comportamento do sistema conversor estático/malha de realimentação na presença de um circuito magnético com material não linear.

\section{MODELAGEM DO DISPOSITIVO ELETROMAGNÉTICO PELO MÉTODO DOS ELEMENTOS FINITOS}

As equações que descrevem o comportamento do dispositivo eletromagnético em 2D podem ser escritas utilizando a formulação do potencial vetor magnético (Sadowski,1993), resultando na equação (1) e na equação (2),

$$
\begin{array}{r}
\frac{\partial}{\partial \mathrm{x}}\left(\nu \frac{\partial A}{\partial \mathrm{x}}\right)+\frac{\partial}{\partial \mathrm{y}}\left(\nu \frac{\partial A}{\partial \mathrm{y}}\right)-\sigma\left(\frac{\partial A}{\partial \mathrm{t}}\right)+ \\
\frac{\mathrm{N} I}{\mathrm{~S}}=-\nu \frac{\partial B_{o y}}{\partial \mathrm{x}}+\nu \frac{\partial B_{o x}}{\partial \mathrm{y}} \\
U=R I+L \frac{\mathrm{d} I}{\mathrm{dt}}+\frac{\mathrm{lN}}{\mathrm{S}} \iint_{\mathrm{S}} \frac{\partial A}{\partial \mathrm{t}} \mathrm{ds}
\end{array}
$$

onde I é a corrente nos enrolamentos do dispositivo, S é a área da bobina, $\mathrm{N}$ é o número de espiras da bobina, l é o comprimento dos fios da bobina, U a diferença de potencial nos terminais da bobina e $\mathrm{L}$ representa a indutância das cabeças de bobina.
A equação (1) e a equação (2) são de difícil abordagem analítica. Portanto, o método dos elementos finitos é utilizado, resultando o sistema matricial dado pelo conjunto de equações (3).

$$
\begin{aligned}
& \mathbf{M A}+\mathbf{N} \frac{\mathrm{d}}{\mathrm{dt}} \mathbf{A}-\mathbf{P I}=\mathbf{D} \\
& \mathbf{Q} \frac{\mathrm{d}}{\mathrm{dt}} \mathbf{A}+\mathbf{R I}+\mathbf{L} \frac{\mathrm{d} \mathbf{I}}{\mathrm{dt}}=\mathbf{U}
\end{aligned}
$$

onde $\mathrm{M}$ é a matriz relacionada à permeabilidade dos meios, A é o potencial vetor nos nós da malha, $\mathrm{N}$ é a matriz relativa à condutividade, $\mathrm{P}$ é a matriz que relaciona a corrente no elemento aos nós do elemento, D é o vetor de excitação induzida por imãs permanentes, Q é o enlace de fluxo nos enrolamentos, L representa as indutâncias nas cabeças de bobina, $\mathrm{R}$ é a resistência dos enrolamentos e I é a corrente nos enrolamentos.

\section{EQUAÇÕES DO CIRCUITO DO CON- VERSOR ESTÁTICO}

A equação diferencial geral de um circuito elétrico conectado a uma estrutura eletromagnética é dada por,

$$
\frac{\mathrm{d} \mathbf{X}}{\mathrm{dt}}=\mathbf{G}_{\mathbf{1}} \mathbf{X}+\mathbf{G}_{\mathbf{2}} \mathbf{E}+\mathbf{G}_{\mathbf{3}} \mathbf{I}
$$

A tensão no dispositivo eletromagnético pode ser calculada pela relação,

$$
\mathbf{U}=\mathbf{G}_{\mathbf{4}} \mathbf{X}+\mathbf{G}_{5} \mathbf{E}+\mathbf{G}_{6} \mathbf{I}
$$

onde X é o vetor das correntes nas indutâncias e as tensões nos capacitores do circuito, E é a matriz da fontes de tensão do circuito exterior e as matrizes $\mathrm{G}_{1}$ a $\mathrm{G}_{6}$ dependem dos elementos e da topologia do conversor.

Os interruptores do circuito do conversor estático são modelados utilizando uma representação por resistores binários. Assim, eles serão considerados como resistores com uma alta resistência quando se encontrarem bloqueados e com uma baixa resistência quando eles estiverem no estado de condução. Por este motivo as matrizes $\mathrm{G}_{1}$ a $\mathrm{G}_{6}$ na equação (4) e equação (5) devem ser recalculadas todas as vezes em que os interruptores mudarem seus estados.

Para representar o interruptor é considerado um modelo lógico que contém uma saída: condução ou bloqueio. A entrada deste modelo é composto por grandezas elétricas, que permitem administrar as comutações (tensão e corrente no interruptor), e as ordens de comando que atuam sobre os terminais de controle. O funcionamento é representado utilizando a Rede de Petri (Roel, 1998), 
onde os lugares representam os estados do interruptor e as transições estão associadas com as condições de comutação que permitem passar de um estado a outro.

\section{ACOPLAMENTO DAS EQUAÇÕES DO DISPOSITIVO ELETROMAGNÉTICO E DO CONVERSOR}

Para a simulação de estruturas eletromagnéticas alimentadas por circuitos exteriores é utilizada uma resolução simultânea das equações do campo e do circuito (figura $1)$.

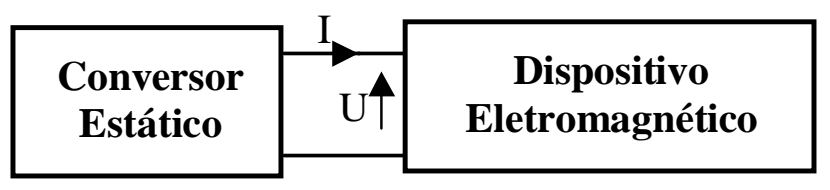

Figura 1: Associação de conversores estáticos com dispositivos eletromagnéticos

A tensão U será comum tanto ao dispositivo quanto ao conversor, sendo a corrente nos enrolamentos do dispositivo eletromagnético desconhecida. Combinando a equação (3), equação (4) e equação (5), obtém-se um sistema matricial global de equações que representa um dispositivo eletromagnético alimentado por conversores estáticos (Kuo-Peng et alii, 1997)

$$
\begin{aligned}
& \mathbf{M A}+\mathbf{N} \frac{\mathrm{d}}{\mathrm{dt}} \mathbf{A}-\mathbf{P I}=\mathbf{D} \\
& \mathbf{Q} \frac{\mathrm{d}}{\mathrm{dt}} \mathbf{A}+\left[\mathbf{R}-\mathbf{G}_{\mathbf{6}}\right] \mathbf{I}+\mathbf{L} \frac{\mathrm{d} \mathbf{I}}{\mathrm{dt}}-\mathbf{G}_{\mathbf{4}} \mathbf{X}=\mathbf{G}_{\mathbf{5}} \mathbf{E} \\
& \frac{\mathrm{d}}{\mathrm{dt}} \mathbf{X}-\mathbf{G}_{\mathbf{1}} \mathbf{X}-\mathbf{G}_{\mathbf{3}} \mathbf{I}=\mathbf{G}_{\mathbf{2}} \mathbf{E}
\end{aligned}
$$

As incógnitas neste sistema de equações são o potencial vetor A nos nós da malha de elementos finitos, a corrente nos enrolamentos do dispositivo eletromagnético I e as variáveis de estado $\mathrm{X}$, que é a tensão nos capacitores e a corrente nos indutores do circuito de alimentação.

O sistema de equações (6) é variável no tempo. Para a sua resolução passo a passo no tempo, utiliza-se o esquema de recorrência de Euler para discretizar as derivadas temporais. Assim, este conjunto de equações no tempo se representa como o sistema de equações discre- $\operatorname{tas}(7)$.

$$
\left\{\begin{array}{c}
M A(\mathrm{t})+\frac{N}{\Delta \mathrm{t}} A(\mathrm{t})-P I(\mathrm{t})=\frac{N}{\Delta \mathrm{t}} A(\mathrm{t}-\Delta \mathrm{t})+D \\
\frac{Q}{\Delta \mathrm{t}} A(\mathrm{t})+\left[R-G_{6}\right] I(\mathrm{t})+\frac{L}{\Delta \mathrm{t}} I(\mathrm{t})-G_{4} X(\mathrm{t})= \\
\frac{Q}{\Delta \mathrm{t}} A(\mathrm{t}-\Delta \mathrm{t})+\frac{L}{\Delta \mathrm{t}} I(\mathrm{t}-\Delta \mathrm{t})+G_{5} E(\mathrm{t}) \\
\frac{1}{\Delta \mathrm{t}} X(\mathrm{t})-G_{1} X(\mathrm{t})-G_{3} I(\mathrm{t})=\frac{1}{\Delta \mathrm{t}} X(\mathrm{t}-\Delta \mathrm{t})+G_{2} E(\mathrm{t})
\end{array}\right.
$$

\section{IMPLEMENTAÇÃO DA MALHA DE CONTROLE}

Os conversores estáticos são sistemas que realizam a função de conversão da energia elétrica de uma forma a outra valendo-se para isto da característica de comutação dos interruptores de potência. O controle desta transferência de energia é obtido ao serem aplicados sinais de controle nestes interruptores afim de modificar os seus tempos de condução.

Na simulação de dispositivos eletromagnéticos, modelados pelo método dos elementos finitos, acoplados a circuitos conversores estáticos é importante considerar o controle dos interruptores de potência. Na maioria dos conversores estáticos as ordens de comando dos interruptores dependem da variável de saída. Assim, é conveniente medir a saída para intervir diretamente na ordenação da comutação dos semicondutores de potência comandados, a fim de que a saída siga uma referência.

O diagrama de blocos que mostra o sistema de controle em laço fechado implementado é mostrado na figura 2. Existem dois blocos principais; o primeiro representa o sistema do acoplamento de conversores estáticos com dispositivos eletromagnéticos e o segundo representa o bloco de controle

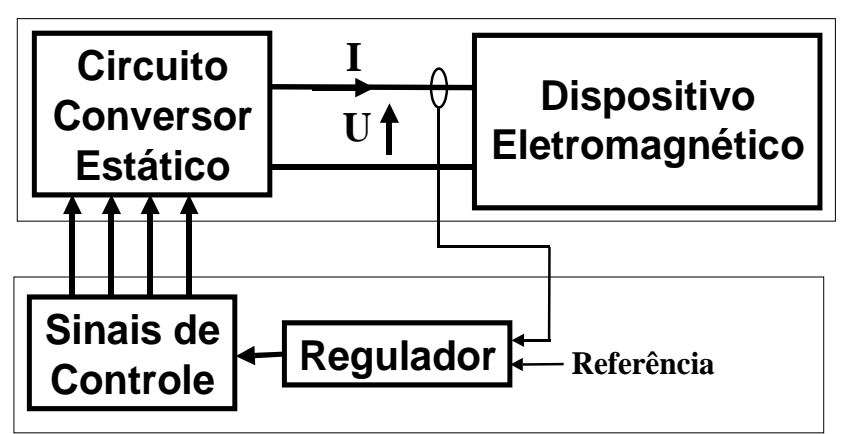

Figura 2: Diagrama de blocos do sistema de controle em laço fechado para a simulação de conversores estáticos acoplados a dispositivos eletromagnéticos 
O intercâmbio de informação entre os dois blocos acontece a cada passo de tempo. Em um sistema de controle de laço aberto, este passo de tempo é calculado a partir da frequiência das fontes e/ou da freqüência de comutação dos interruptores. Isto assegura que não haverá perda de informação acerca das comutações devido a uma escolha errada do passo de tempo. Quando o sistema opera em laço fechado, os sinais de controle da comutação são gerados automaticamente de acordo com o valor da variável a ser controlada e a lei de controle. Neste caso, o passo de tempo depende da dinâmica do controlador e do circuito. A grandeza a ser controlada, tensão e/ou corrente em um componente, é obtida utilizando um resistor como sensor. Esta operação consiste em posicionar um resistor com baixa resistência em série com o componente cuja corrente será controlada ou um resistor com alta resistência em paralelo com o componente de quem se deseja controlar a tensão.

A malha de controle implementada consiste em medir a grandeza a ser controlada (tensão e/ou corrente de um componente) a cada passo de tempo e compará-la com um sinal de referência imposta pelo usuário.

A cada passo de cálculo o programa verifica a existência de comutações nos interruptores do circuito de alimentação e determina os instantes de condução e bloqueio (Roel et alii, 2000). A figura 3 mostra o fluxograma da metodologia implementada.

\section{APLICAÇÃO}

Para verificar a metodologia proposta foi implementado um inversor em ponte completa com um indutor saturável como carga não linear. Para a malha de controle, utilizou-se um controle do tipo robusto, o controle por modos deslizantes - "Sliding Mode Control (SMC)" - conforme a metodologia de projeto e a implementação em freqüência fixa realizada por Batistela e Perin (1995), teoricamente baseado em Slotine (1991). Jung e Tzou (1996) apresentam uma estrategia de implementação deste controle bastante semelhante.

\subsection{O dispositivo eletromagnético}

Como elemento não linear é utilizado um núcleo do tipo pote, figura 4 , sem entreferro.

O dispositivo eletromagnético é simulado utilizando o programa EFCAD (Bastos, 1992) em duas dimensões com geometria axi-simétrica. O mapa de campo para este dispositivo, assim como o desenho da estrutura são apresentadas na figura 5 .

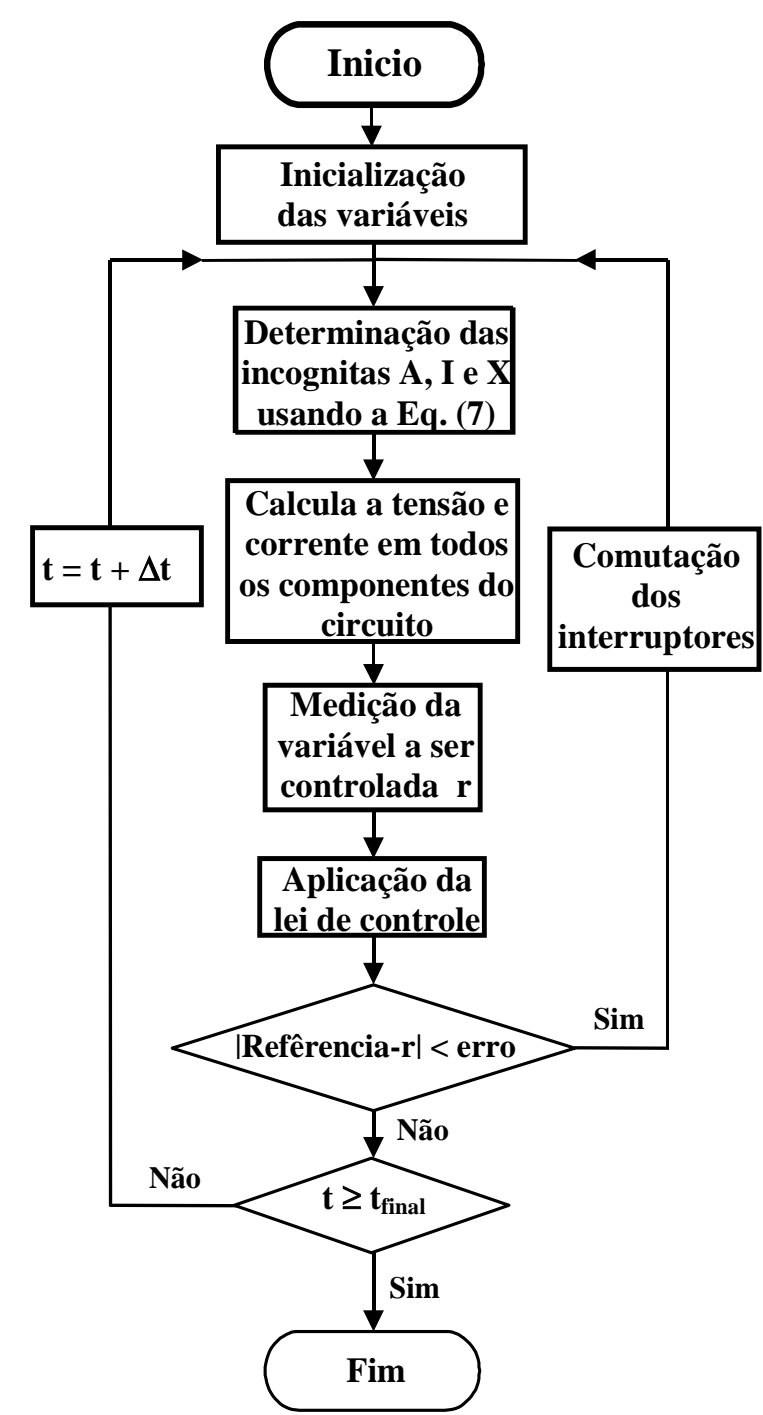

Figura 3: Fluxograma da simulação de dispositivos eletromagnéticos alimentados por conversores estáticos com malha de controle

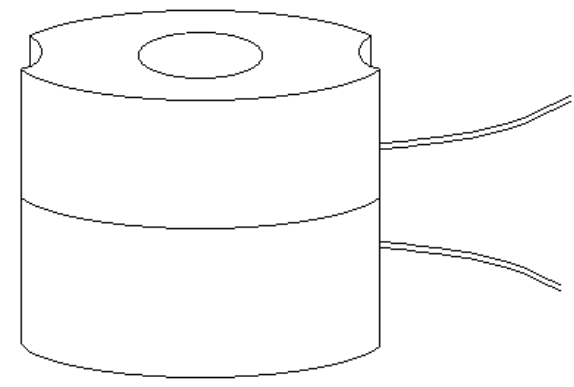

Figura 4: Geometria do núcleo pote 


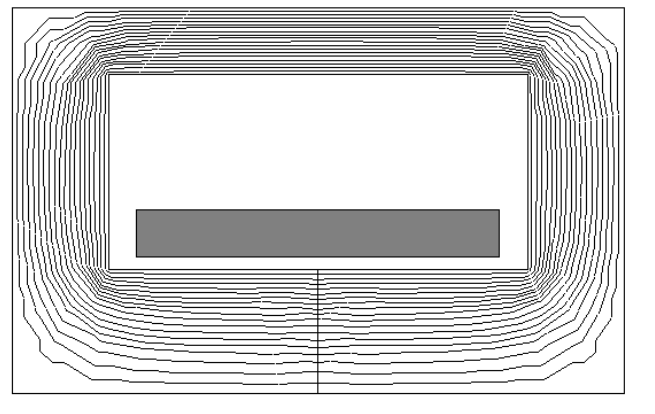

ๆ

Figura 5: Modelo do dispositivo eletromagnético em 2D

A característica não linear do dispositivo eletromagnético é considerada utilizando a curva $\mathrm{BH}$ da figura 6 .

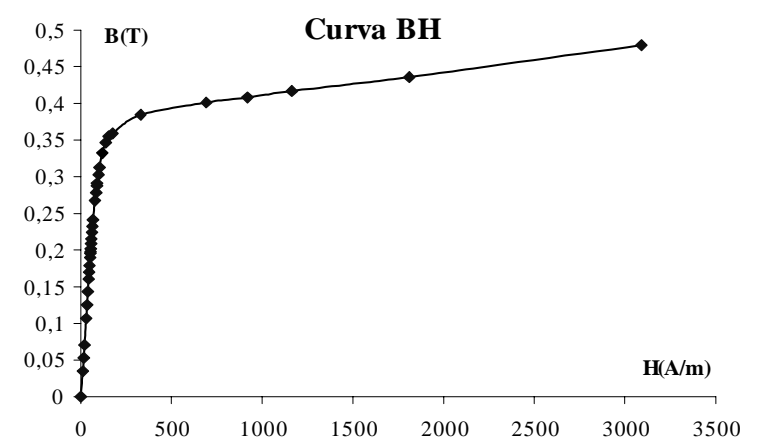

Figura 6: Curva BH para o núcleo de ferrite tipo pote

\subsection{0 inversor de tensão e seu controle}

O conversor estático implementado e simulado está mostrado na figura 7. É um inversor monofásico em ponte completa e filtro LC, tendo um indutor saturado como carga, onde $\mathrm{E}$ é a tensão de entrada $\mathrm{CC}, \mathrm{S}_{i}$ são os interruptores de potência comandados, $\mathrm{D}_{i}$ são os diodos de roda livre, $\mathrm{C}_{o}$ e L são o capacitor e indutor de filtro respectivamente, $\mathrm{L}_{o}$ é o indutor de carga não linear, $\mathrm{V}_{a b}$ é a tensão entre os pontos "a" e "b", $\mathrm{i}_{o}$ é a corrente na carga e $\mathrm{V}_{c o}$ é a tensão de saída do inversor.

As equações que representam o modelo da operação do inversor sem carga são escritas no sistema matricial (8) na forma de modelo de espaço de estado.

$\frac{\mathrm{d}}{\mathrm{dt}}\left[\begin{array}{l}V c o \\ i_{L}\end{array}\right]=\left[\begin{array}{ll}0 & 1 / C o \\ -1 / L & 0\end{array}\right]\left[\begin{array}{l}V c o \\ I_{L}\end{array}\right]+\left[\begin{array}{l}0 \\ 1 / L\end{array}\right]\left[V_{a b}\right]$

O sistema matricial (8) é contínuo por partes no tempo: a tensão Vab muda o seu valor de $\mathrm{E}$ para $-\mathrm{E}$, e vice e versa, dependendo do estado de comando dos interruptores. Assim, Vab será igual a $\mathrm{uE}$, dependendo da ordem de comando u ter valor $1 \mathrm{ou}-1$.

O objetivo da malha de controle é fazer com que a tensão de saída $\mathrm{V}_{c o}$ siga um sinal de referência senoidal, sendo que a carga do inversor é não linear.

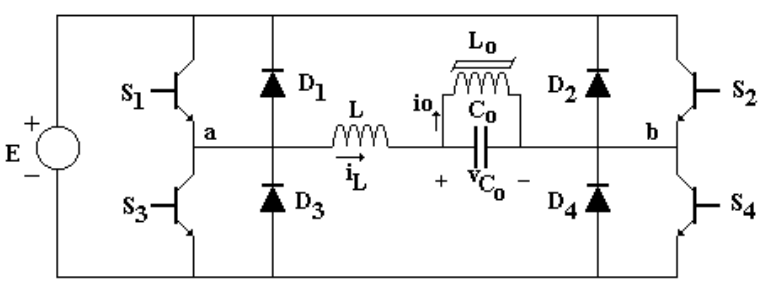

Figura 7: Inversor ponte completa

As variáveis de estado de um sistema, ao sofrerem uma excitação evoluem ao longo do tempo segundo uma lei natural própria até um ponto de equilíbrio ou para a instabilidade. Uma maneira usual de representar a evolução de um destes sistemas se constitui em determinar a trajetória do sistema no espaço de estado. O SMC corresponde a um caminho o qual restringe as trajetórias das variáveis no espaço n-dimensional de estado. Isto é, o SMC obriga a trajetória descrita pela evolução do sistema no tempo a seguir posicionamentos instantâneos pré-determinados no espaço n-dimensional. Este caminho pode ser uma reta se for de primeira ordem ou um plano se for de segunda ordem. Quando o sistema descreve sua trajetória de estado de forma coincidente com o caminho de controle (Sliding control), o sistema está sofrendo a atuação efetiva do controle.

Segundo a implementação de Batistela e Perin (1995) para este tipo de estrutura de eletrônica de potência, controlando somente a tensão de saída do inversor, a superfície de deslizamento pode ser composta pelo erro e sua derivada. O erro instantâneo entre a referência e a saída do sistema é dado pela equação (9). A superfície de deslizamento de segunda ordem é dada pela equação (10), a qual descreve geometricamente um plano formado pelo erro e sua derivada, representado pela superfície $\mathrm{s}(\varepsilon, \mathrm{t})$ na figura 8 .

$$
\begin{gathered}
\varepsilon(t)=V_{\text {ref }}(t)-V_{\text {saída }}(t) \\
s(\varepsilon, t)=\frac{\mathrm{d} \varepsilon}{\mathrm{dt}}+\lambda \varepsilon
\end{gathered}
$$

A variável $\mathrm{s}(\varepsilon, \mathrm{t})$ determina o valor da variável de atuação u. Para $\mathrm{s}(\varepsilon, \mathrm{t})>0, \mathrm{u}=-1$ e para $\mathrm{s}(\varepsilon, \mathrm{t})<0, \mathrm{u}=1$. $\mathrm{A}$ variação da estrutura controlada pelo sinal $\mathrm{s}(\varepsilon, \mathrm{t})$ 


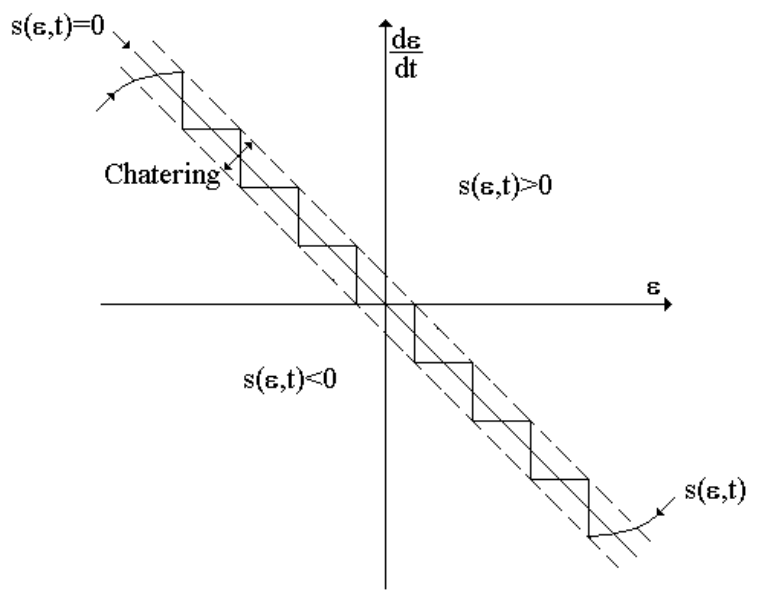

Figura 8: Plano de erro (Plano s)

faz com que o sistema tenda a permanecer na reta $\mathrm{s}(\varepsilon, \mathrm{t})=0$, tendendo necessariamente ao valor de equilíbrio $(\varepsilon, \mathrm{d} \varepsilon / \mathrm{dt})=(0,0)$. Diz-se que o sistema está no modo deslizante quando permanece na reta $\mathrm{s}(\varepsilon, \mathrm{t})=0$. A permanência do sistema deslizando sobre esta reta torna o sistema estável e, na situação $(\varepsilon, \mathrm{d} \varepsilon / \mathrm{dt})=(0,0)$, a saída está seguindo a referência.

A condição de existência do modo deslizante, dada pela equação (11), que deve ser satisfeita pela mudança da variável de atuação u para que o sistema permaneça no ponto de equilíbrio, garante também a robustez do sistema. O esquema básico da implementação do controle está apresentado na figura 9.

$$
\left\{\begin{array}{c}
\lim _{s \rightarrow 0^{+}} \frac{\mathrm{d} s}{\mathrm{dt}}<0 \\
\lim _{s \rightarrow 0^{-}} \frac{\mathrm{d} s}{\mathrm{dt}}>0
\end{array}=s \frac{\mathrm{d} s}{\mathrm{dt}}<0\right.
$$

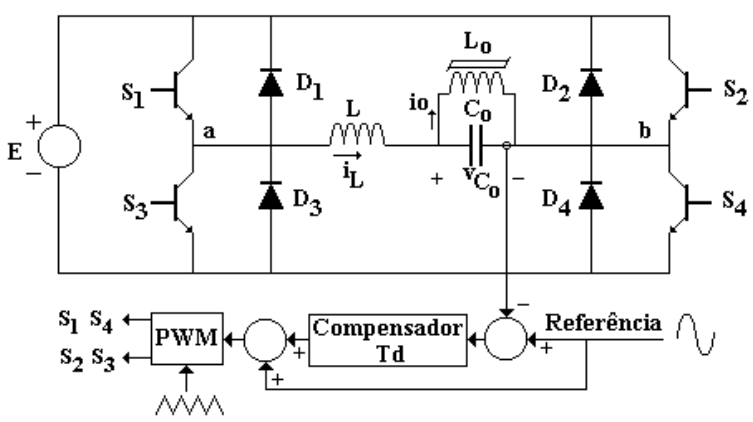

Figura 9: Diagrama esquemático do inversor e o bloco de controle

O diagrama de blocos do controle em laço fechado implementado é mostrado na figura 10. A saída do inversor $\left(\mathrm{V}_{c o}\right)$ é comparada com uma referência e o erro $\varepsilon$, obtido desta comparação, é aplicado ao controle por modos deslizantes com o propósito de atingir a situação $\mathrm{s}(\varepsilon, \mathrm{t})=0$. A característica de freqüência fixa é dada por uma estratégia de comutação PWM (Batistela e Perin, 1995).

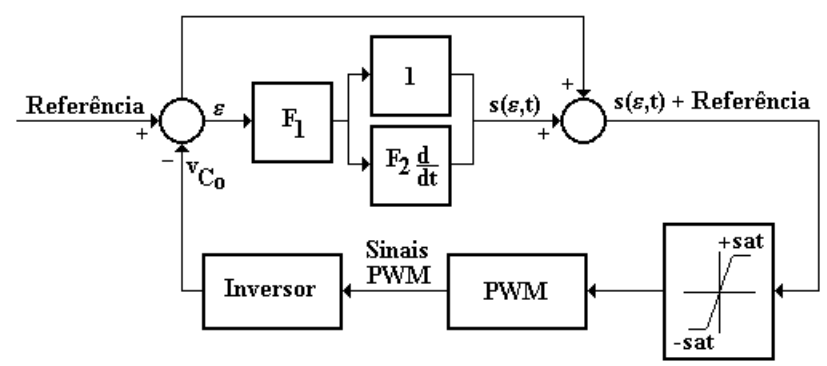

Figura 10: Diagrama de blocos do controle SMC implementado

\section{RESULTADOS}

$\mathrm{Na}$ implementação experimental do inversor de tensão foram utilizados os seguintes parâmetros:

Tensão de alimentação CC : 25 volts,

Capacitor do filtro de saída: $24 \mu \mathrm{F}$,

Indutor de filtro de saída : $800 \mu \mathrm{H}$,

Tensão de saída do inversor : 2.25 volts,

Freqüência da tensão de saída : $50 \mathrm{~Hz}$.

A forma de onda da referência é sinusoidal com uma freqüência de $50 \mathrm{~Hz}$ e uma amplitude de 5.5 volts. Os ganhos $\mathrm{F}_{1}$ e $\mathrm{F}_{2}$ do controlador (figura 10) tem valores de 37.037 e $7.5 \times 10^{-6}$ respectivamente. A freqüência fixa é dada pela comparação do sinal $\mathrm{s}(\varepsilon, \mathrm{t})+$ Referência com um sinal triangular de 8 volts de amplitude e freqüência de $30 \mathrm{KHz}$.

O resultado experimental da corrente no indutor como carga não-linear é mostrado na figura 11 juntamente com a corrente obtida na simulação do sistema. Quando a saturação não é alcançada, a corrente é praticamente proporcional ao fluxo e a curva $\mathrm{BH}$ se encontra na região linear. Quando a saturação acontece, a corrente cresce muito mais rapidamente que o fluxo, isto é, grandes mudanças no valor da corrente não produzem grandes variações na amplitude do fluxo no ferrite.

Pode-se observar que existem diferenças entre as curvas medida e simulada, as quais podem ser produzidas pela falta de exatidão na característica $\mathrm{BH}$ utilizada no cálculo. Pequenas imprecisões na obtenção da curva BH 


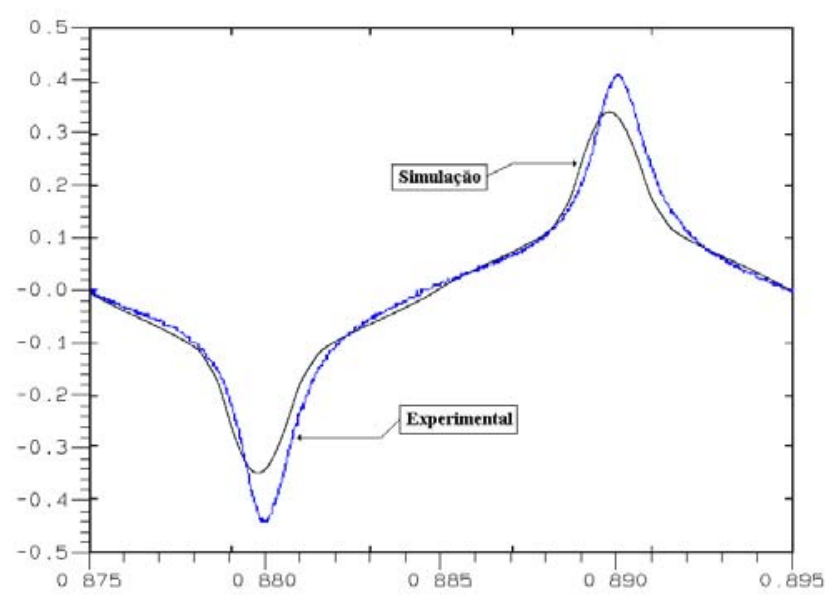

Figura 11: Corrente no indutor de carga não-linear. Resultado experimental e simulado

experimental nesta região de saturação produzem significativas diferenças na curva da corrente simulada, pois o valor da indução no dispositivo simulado é dado pela tensão imposta no mesmo.

Entretanto, devido à forte não linearidade presente na região de saturação, caso o modelo utilizado no programa de simulação não fosse próximo à realidade, facilmente o ponto de operação do sistema simulado em malha fechada produziria valores de corrente de saturação máxima bem distantes do valor real.

\section{CONCLUSÃO}

Foi apresentado um método de simulação de dispositivos eletromagnéticos acoplados a conversores estáticos considerando a malha de realimentação. Como exemplo de aplicação desta metodologia foi utilizado um inversor de tensão em ponte completa, controlado em malha fechada, com carga não-linear, onde o elemento não-linear é um indutor modelado pelo método de elementos finitos em 2D. Ainda que esta aplicação não seja de interesse prático, o exemplo serve para mostrar o funcionamento da metodologia proposta.

A modelagem de dispositivos eletromagnéticos pelo método de elementos finitos apresenta vantagens frente a outras ferramentas de simulação. O uso de métodos numéricos possibilita o análise de dispositivos com geometrias complexas, onde uma representação analítica é difícil. Com o uso dos métodos numéricos, pode-se descrever de modo mais preciso o comportamento destes dispositivos.
Os resultados de simulação obtidos mostram que o programa computacional do acoplamento de conversores estáticos e sua malha de controle com dispositivos eletromagnéticos operando na região não- linear têm uma boa concordância com os obtidos experimentalmente.

Este método oferece uma poderosa ferramenta para o projeto e análise de sistemas compostos por dispositivos eletromagnéticos não-lineares acoplados a conversores estáticos controlados em malha fechada.

\section{REFERÊNCIAS}

Balabanian, N. \& T. Bickart (1969). Electrical Network Theory. John Willey \& Sons, Inc.

Bastos, J.P.A. (1992). Eletromagnetismo e Cálculo de Campos. 2a ed. Editora da UFSC, Florianópolis.

Batistela, N.J. \& A. J. Perin (1995). A Fixed Frequency Sliding Mode Control For Voltage Source Inverter. Anais do $3^{\circ}$ Congresso Brasileiro de Eletrônica de Potência COBEP'95, São Paulo S.P., pp. 229-234.

Jung. S. L. \& Y.Y. Tzou (1996). Discrete Sliding-Mode Control of a PWM Inverter for Sinusoidal Output Waveform Synthesis with Optimal Sliding Curve. IEEE Transactions on Power Electronics (Jul.), Vol 11, No. 4, pp. 567-577.

Kuo-Peng, P., N. Sadowski, J.P.A. Bastos, R. Carlson, N.J. Batistela, M. Lajoie-Mazenc (1997). A General Method for Coupling Static Converter with Electromagnetics Structures. IEEE Transactions on Magnetics (Mar.), Vol 33, No. 2, pp. 2004-2009.

Roel, J.L. (1998). Análise do Conjunto ConversorDispositivo Eletromagnético: Contribuição à Criação de Biblioteca de Controle. Dissertação de Mestrado, Universidade Federal de Santa Catarina, UFSC/CTC/EEL/GRUCAD, Florianópolis - SC.

Roel, J.L., N. Sadowski, P. Kuo-Peng, N.J. Batistela, J.P.A. Bastos (2000). Coupling Static Converter with Control Loop and Non-Linear Electromagnetic Devices. Proc. Of the $9^{\text {th }}$ Biennial IEEECEFC'2000, Milwaukee, Wisconsin, USA, p. 102.

Sadowski, N. (1993). Modélisation des Machines Électriques à Partir de la Résolution des Équations du Champ en Tenant Compte du Mouvement et du Circuit d'Alimentation (Logiciel EFCAD). Thèse de doctorat, I.N.P.T., Toulouse, França.

Slotine, J.J. (1991). Applied Nonlinear Control. Prentice Hall, New Jersey. 